\title{
Characterizing Positive Definite Matrices with t-norms
}

\author{
J.Recasens \\ Dept. Tecnologia de l'Arquitectura \\ ETS Arquitectura del Vallès \\ Universitat Politècnica de Catalunya \\ C. Pere Serra 1-15 \\ j.recasens@upc.edu
}

\begin{abstract}
In this work symmetric positive definite matrices with non-negative entries will be characterized by using the Yager's family of tnorms. It can always be assumed that such an $n \times n$ matrix corresponds to a reflexive and symmetric fuzzy relation $A$ on a set of cardinality $n$ and then $A$ is positive definite if and only if it is transitive with respect to a specific t-norm $T_{\lambda}$ of Yager's family with $\lambda$ depending on $n$. The result will be applied to give alternative proofs of the following two facts.
\end{abstract}

- Every min-indistinguishability operator separating points on a finite set has a positive definite matrix.

- Every ultrametric on a finite set is Euclidean.

Keywords: Positive definite matrix, Tolerance relation, Transitivity, Indistinguishability operator, Yager's family of t-norms, Euclidean metric space.

\section{Introduction}

Positive definite matrices appear in many branches of Mathematics such as in the study of quadratic forms, optimization problems and classification of quadric (hyper)-surfaces; but what is more important in Artificial Intelligence is that in linear algebra the matrix associated to an inner product of a vector space is positive definite. In this case, an Euclidean distance can be defined on this space. In many AI problems, in particular in machine learning, vision or cluster analysis, a distance on a universe $X$ is needed [7]. In these cases, the use of kernels on $X$ allows us to define an Euclidean-like distance $d$ on $X$ with all its good properties $[6,7]$.
There are many classical characterizations of positive definite matrices (we recall the main important two in Proposition 1.6).

Surprisingly, the use of t-norms has been proved a good tool for studying these matrices as it can be found in $[5,11,9]$.

In $[5,11]$ it has been proved that if a reflexive and symmetric fuzzy relation is positive semi-definite, then it is transitive with respect to the continuous Archimedean t-norm $T_{\text {arccos }}$ having the function arccos as additive generator; in [11], it is also proved that it is also transitive with respect to the continuous Archimedean tnorm $T_{\sqrt{1-x}}$ having the function $t(x)=\sqrt{1-x}$ as additive generator. The reciprocal of both assertions is not true but in [9] the following two characterizations in which an additional embedding property of metric spaces is involved can be found.

Proposition 1.1. A tolerance relation $E=\left(x_{i j}\right)$ on a set $X=\left\{x_{1}, x_{2}, \ldots, x_{n}\right\}$ of finite cardinality $n$ is positive definite if and only if it is a $T_{\sqrt{1-x}}$-indistinguishability operator and $X$ with the distance $d\left(x_{i}, x_{j}\right)=\sqrt{2} \sqrt{1-x_{i j}}, 1 \leq i, j \leq n$, is embeddable into $\mathbb{R}^{n}$ in such a way that the images of the points of $X$ lie on the hypersphere $\mathbb{S}^{n-1}$.

Proposition 1.2. A tolerance relation $E$ on a set $X=\left\{x_{1}, x_{2}, \ldots, x_{n}\right\}$ of finite cardinality is positive definite if and only if it is a $T_{\arccos }$-indistinguishability operator and $X$ with the distance $d\left(x_{i}, x_{j}\right)=\arccos x_{i j}$, $1 \leq i, j \leq n$ can be mapped isometrically into $\mathbb{S}^{n-1}$ with the spherical metric.

In the current work symmetric positive definite matrices with non-negative entries will be characterized thanks to the Yager's family of t-norms. More precisely, after proving that the entries of a positive definite matrix $A$ with non-negative values can be assumed to be in the unit interval, it will be characterized as being transitive with respect to a specific t-norm $T_{\lambda}$ of the Yager's family where $\lambda$ depends on the order of $A$ (Proposition 3.5). 
The rest of this section contains the basic definitions and properties of indistinguishability operator, tolerance relation and positive definite matrix.

Definition 1.3. [8] Let $T$ be a t-norm and $X$ a set. A fuzzy relation $E$ on $X$ is a $T$-indistinguishability operator if and only if for all $x, y, z \in X$

- $E(x, x)=1$ (Reflexivity)

- $E(x, y)=E(y, x)$ (Symmetry)

- $T(E(x, y), E(y, z)) \leq E(x, z)$ (T-transitivity).

If $E(x, y)=1$ if and only if $x=y$, then $E$ separates points.

Definition 1.4. A reflexive and symmetric fuzzy relation $E$ on $X$ is called a proximity or tolerance relation.

If $X=\left\{x_{1}, x_{2}, \ldots, x_{n}\right\}$ is a finite set, then a tolerance relation $E$ can be identified with the matrix $E=\left(x_{i j}\right)_{i, j=1,2, \ldots, n}$ where $x_{i j}=E\left(x_{i}, x_{j}\right)$.

Definition 1.5. A symmetric $n \times n$ real matrix $A$ is positive definite if $\vec{u}^{t} A \vec{u}>0$ for every non-zero vector $\vec{u} \in \mathbb{R}^{n}$ and positive semi-definite if $\vec{u}^{t} A \vec{u} \geq 0$.

There are many characterizations of positive definiteness. The next proposition recalls a couple of them and can be found in any book of linear algebra.

Proposition 1.6. Let $A$ be a symmetric $n \times n$ real matrix. The following statements are equivalent.

a) $A$ is positive definite.

b) All eigenvalues of $A$ are positive.

c) All principal minors of $A$ are positive, where the $k$-th principal minor of a matrix $A$ is the determinant of its upper-left $k \times k$ sub-matrix.

In the proof of Proposition 3.2 we will need the following result.

Proposition 1.7. The product of positive definite matrices is a positive definite matrix.

\section{$2 \quad$ Needed Results}

In this section important known results will be presented that will be used in Section 3 to our characterization.

Definition 2.1. Let $X$ be a set. A mapping $d: X \times$ $X \rightarrow[0, \infty]$ is a pseudodistance or pseudometric if and only if for all $x, y, z \in X$

- $d(x, x)=0$

- $d(x, y)=d(y, x)$
- $d(x, y)+d(y, z)) \geq d(x, z)$

If $d(x, y)=0$ if and only if $x=y$, then $d$ is a distance or metric on $X$.

The next proposition shows the way indistinguishability operators are related to distances for continuous Archimedean t-norms.

Proposition 2.2. [12] Let $T$ be a continuous Archimedean $t$-norm, $t$ an additive generator of $T$ and $X$ a set. A fuzzy relation $E$ on $X$ is a $T$-indistinguishability operator if and only if $t \circ E$ is a pseudodistance on $X$. $E$ separates points if and only if $t(E)$ is a distance.

Lemma 2.3. Let $T$ be a $t$-norm, $X$ a set, $x, y \in X$ and $E$ a $T$-indistinguishability operator on $X$. If $E(x, y)=$ 1 , then for all $z \in X, E(x, z)=E(y, z)$ (i.e.: the columns of $E$ corresponding to $x$ and $y$ coincide).

Proof. Since $E$ is $T$-transitive,

$$
E(y, z)=T(E(x, y), E(y, z)) \leq E(x, z) .
$$

Similarly, $E(x, z) \leq E(y, z)$.

The next result states that a positive definite $T$ indistinguishability operator must separate points.

Proposition 2.4. Let $T$ be a t-norm, $X$ a set and $E$ a $T$-indistinguishability operator on $X$ which is positive definite. Then E separates points.

Proof. If $E$ does not separate points, then there exists $x, y \in X(x \neq y)$ with $E(x, y)=1$ and thanks to the previous Lemma 2.3, $E$ has two equal columns. Therefore, its determinant is 0 and cannot be positive definite.

Definition 2.5. If a metric space $(S, d)$ is isometrically embeddable in an Euclidean space, we will say that $d$ is Euclidean.

Corollary 2.7 provides a characterization of metric spaces isometrically embeddable into an Euclidean space. This result together with the ones in [2] recalled below will be the cornerstone to prove the characterization of positive definite matrices with the use of the Yager's family of t-norms.

Proposition 2.6. [10] Let $(S, d), S=\left\{x_{0}, x_{1}, \ldots, x_{n}\right\}$, be a finite metric space of $n+1$ points. Then $d$ is Euclidean if and only if the matrix $A$ with entries $x_{i j}=$ $\frac{1}{2}\left(d_{0 i}^{2}+d_{0 j}^{2}-d_{i j}^{2}\right), i, j=1, \ldots, n$ where $d_{i j}$ stands for $d\left(x_{i}, x_{j}\right)$ is positive semi-definite.

If $A=\left(x_{i j}\right)$ is a reflexive and symmetric fuzzy relation, its associated $n \times n$ matrix has ones in its diagonal, so that for all $i=1,2, \ldots, n$, we have

$$
1=x_{i i}=\frac{d_{0 i}^{2}+d_{0 i}^{2}-d_{i i}^{2}}{2}=\frac{2 d_{0 i}^{2}}{2}=d_{0 i}^{2}
$$


and hence

$$
d_{0 i}=1 \text {. }
$$

Moreover, from

$$
x_{i j}=\frac{d_{0 i}^{2}+d_{0 j}^{2}-d_{i j}^{2}}{2}=\frac{2-d_{i j}^{2}}{2}
$$

we get

$$
d_{i j}=\sqrt{2} \sqrt{1-x_{i j}} \text { for all } 1 \leq i, j \leq n .
$$

Corollary 2.7. Let $E=\left(x_{i j}\right)_{i, j=1,2, \ldots, n}$ be a tolerance relation on a finite set $X=\left\{x_{1}, \ldots x_{n}\right\}$. Then $E$ is a positive definite matrix if and only if $d: X \cup\left\{x_{0}\right\} \times X \cup$ $\left\{x_{0}\right\} \rightarrow[0,1]$ defined for all $x_{i}, x_{j} \in X$ by $d\left(x_{i}, x_{j}\right)=$ $\sqrt{2} \sqrt{1-x_{i j}}$ if $1 \leq i, j \leq n, d\left(x_{0}, x_{i}\right)=1$ if $i \neq 0$ and $d\left(x_{0}, x_{0}\right)=0$ is a metric and $X \cup\left\{x_{0}\right\}$ is isometrically embeddable in an Euclidean space.

If we restrict the distance defined in the last corollary to $X$, we obtain the following result.

Corollary 2.8. If $E=\left(x_{i j}\right)_{i, j=1,2, \ldots, n}$ is a positive definite tolerance relation on a finite set $X=$ $\left\{x_{1}, \ldots x_{n}\right\}$, then $d: X \times X \rightarrow[0,1]$ defined for all $x_{i}, x_{j} \in X$ by $d\left(x_{i}, x_{j}\right)=\sqrt{1-x_{i j}}$ is a metric and $X$ is isometrically embeddable in an Euclidean space.

The reciprocal of the previous corollary is not true as is shown int the next counterexample.

Counterexample 2.9. The determinant of the matrix

$$
A=\left(\begin{array}{ccc}
1 & 0.1 & 0.8 \\
0.1 & 1 & 0.7 \\
0.8 & 0.7 & 1
\end{array}\right)
$$

is $\operatorname{det}(A)=-0.028<0$ and hence $A$ is not positive definite but $\sqrt{1-0.1}=0.949, \sqrt{1-0.8}=0.447$ and $\sqrt{1-0.7}=0.548$ are the sides of a triangle and hence determine an Euclidean distance.

The next proposition provides a relationship between distances and $\mathrm{L}$-indistinguishability operators where $\mathrm{E}$ stands for the Eukasiewicz t-norm.

Proposition 2.10. [3, 8] Let $E: X \times X \rightarrow[0,1]$ be a tolerance relation on a set $X . E$ is an $E$ indistinguishability operator if and only if $1-E$ is a pseudometric on $X$.

Hence, every distance can be written in the form $1-E$ where $E$ is an $\mathrm{E}$-indistinguishability operator (separating points).

The last result that will be needed in Section 3 is due to M. Deza and H. Maehara in [2] where the authors study the values $c$ for which, given a set $X$ of finite cardinality $n$ and a distance $d$ on $X$, the power of $d$ to $c\left(d^{c}\right)$ is Euclidean. In particular they prove that for a set of cardinality 6 , the greatest value $c_{6}$ of $c$ is $\frac{1}{2} \log _{2} \frac{3}{2} \sim 0.2924$. Of course, as the cardinality $n$ of the set increases, the corresponding greatest value $c_{n}$ decreases.

Thanks to a result by Blumenthal [1], $c_{4}=\frac{1}{2}$.

\section{The Characterization}

In this section we will characterize positive definite matrices with non-negative entries with the use of the Yager's family of t-norms.

First, let us prove (Proposition 3.2) that we can restrict the characterization to tolerance relations (i.e.: the matrix $A=\left(a_{i j}\right)$ satisfies $a_{i i}=1$ and $0 \leq a_{i j}=$ $\left.a_{j i} \leq 1\right)$.

Lemma 3.1. Let $A=\left(a_{i j}\right) 1 \leq i, j \leq n$ be a symmetric positive definite matrix. Then for all $1 \leq i, j \leq n$

a) $a_{i i}>0$.

b) $a_{i i} \cdot a_{j j} \geq a_{i j}^{2}$.

Proof. a) Trivial thanks to Proposition 1.6c).

b)

$$
\left|\begin{array}{ll}
a_{i i} & a_{i j} \\
a_{j i} & a_{j j}
\end{array}\right|=a_{i i} \cdot a_{j j}-a_{i j} \cdot a_{j i}=a_{i i} \cdot a_{j j}-a_{i j}^{2} \geq 0 .
$$

Proposition 3.2. Let $A=\left(a_{i j}\right) 1 \leq i, j \leq n$ be $a$ symmetric matrix with non-negative entries and $a_{i i}>$ 0 for all $1 \leq i \leq n$ and $D=\operatorname{diag}\left(\frac{1}{\sqrt{a_{11}}}, \frac{1}{\sqrt{a_{22}}} \ldots, \frac{1}{\sqrt{a_{n n}}}\right)$ be the $n \times n$ matrix with diagonal $\frac{1}{\sqrt{a_{11}}}, \frac{1}{\sqrt{a_{22}}} \ldots, \frac{1}{\sqrt{a_{n n}}}$ an zeros otherwise. Then $E=D \cdot A \cdot D$ is a positive definite tolerance relation if and only if $A$ is a positive definite matrix.

Proof. $E=\left(e_{i j}\right), 1 \leq i, j \leq n$ is the matrix with entries $e_{i j}=\frac{a_{i j}}{\sqrt{a_{i i}} \cdot \sqrt{a_{j j}}}$.

a) For $1 \leq i \leq n, e_{i i}=\frac{a_{i i}}{\sqrt{a_{i i}} \cdot \sqrt{a_{i i}}}=1$ and $E$ is reflexive.

b) Thanks to the previous lemma,

$$
1 \geq e_{i j}=\frac{a_{i j}}{\sqrt{a_{i i}} \cdot \sqrt{a_{j j}}} \geq 0
$$

If $A$ is positive definite, then $E$, being the product of positive definite matrices also is positive definite (Proposition 1.7) and thanks to Lemma 3.1b), $0 \leq e_{i j} \leq 1$.

Reciprocally, $A=D^{-1} \cdot E \cdot D^{-1}$ which is positive definite if $E$ is. 
Yager's family of t-norms is defined as follows.

Example 3.3. [4] The Yager's family of continuous Archimedean t-norms $\left(T_{\lambda}\right)_{\lambda \in(0, \infty)}$ is defined for all $x, y \in[0,1]$ by

$$
T_{\lambda}(x, y)=\max \left(\left(1-(1-x)^{\lambda}+(1-y)^{\lambda}\right)^{\frac{1}{\lambda}}, 0\right) .
$$

$t_{\lambda}$ defined by $t_{\lambda}(x)=(1-x)^{\lambda}$ for all $x \in[0,1]$ is an additive generator of $T_{\lambda}$.

\section{Properties 3.4.}

- All t-norms of Yager's family are non-strict.

- If $\lambda>\mu$, then $T_{\lambda}(x, y) \geq T_{\mu}(x, y)$ for all $x, y \in$ $[0,1]$

- If $\lambda=1$, then we recover the Eukasiewicz t-norm $E$ and $t_{1}(x)=1-x$ is an additive generator of $E$.

- $\lim _{\lambda \rightarrow \infty} T_{\lambda}(x, y)=\min (x, y)$ for all $x, y \in[0,1]$.

Now applying the results of the previous section we get the following characterization of positive definite matrices.

Proposition 3.5. Characterization of Positive Definite Matrices. For a given natural number $n$, let $c_{n}$ be the greatest value satisfying that for every distance $d$ on any finite set of cardinality $n, d^{c_{n}}$ is an Euclidean distance. Then a fuzzy relation $E: X \times X \rightarrow$ $[0,1]$ on a set $X=\left\{x_{1}, x_{2}, \ldots, x_{n}\right\}$ of cardinality $n$ is a $T_{\frac{1}{2 c_{n+1}}}$-indistinguishability operator separating points if and only if it is positive definite.

Proof. If $E$ is a $T_{\frac{1}{2 c_{n+1}}}$-indistinguishability operator separating points, then, thanks to Proposition 2.2, (1$E)^{\frac{1}{2 c_{n+1}}}\left(\right.$ and also $d=(2(1-E))^{\frac{1}{2 c_{n+1}}}$ ) is a distance on $X$ that can be extended to a distance $d^{\prime}$ on $X \cup\left\{x_{0}\right\}$ by $d^{\prime}\left(x_{0}, x_{i}\right)=1$ for all $1 \leq i \leq n$ and $d^{\prime}\left(x_{0}, x_{0}\right)=$ 0 . Then $D=d^{\prime c_{n+1}}$ is: $D\left(x_{i}, x_{j}\right)=\sqrt{2} \sqrt{1-x_{i j}}$ if $1 \leq i, j \leq n, D\left(x_{0}, x_{i}\right)=1$ if $i \neq 0$ and $D\left(x_{0}, x_{0}\right)=$ 0 . $D$ is Euclidean and by Corollary $2.7 E$ is positive definite.

$c_{n}$ is not known except for very few values (for $n=$ $2,3,4,6$, the corresponding $c_{n}$ are $c_{2}=\infty, c_{3}=1$, $c_{4}=\frac{1}{2}, c_{6}=\frac{1}{2} \log _{2} \frac{3}{2} \sim 0.2924$ [2]) but in [2] a lower bound $k_{n}$ for $c_{n}$ is given. Namely, $k_{n}=\frac{1}{2 n} \log _{2} \mathrm{e} \sim$ $\frac{0.7213}{n}$. Therefore we have the following result.

Proposition 3.6. If a tolerance relation $E: X \times X \rightarrow$ $[0,1]$ on a set $X$ of cardinality $n$ is $T_{\frac{n+1}{\log _{2} e}}$-transitive, then it is positive semi-definite.
Also in [2] it is conjectured that the value of $c_{n}$ is

$$
c_{n}= \begin{cases}\frac{1}{2} \log _{2}\left(\frac{n}{n-2}\right) & \text { if } n \text { is even } \\ \frac{1}{2} \log _{2}\left(\frac{n^{2}-1}{n^{2}-2 n-1}\right) & \text { if } n \text { is odd. }\end{cases}
$$

From this we can conjecture the following.

\section{Conjecture 3.7 .}

- A fuzzy relation $E: X \times X \rightarrow[0,1]$ on a set $X$ of even cardinality $n$ is a $T_{\frac{1}{\log _{2}\left(\frac{n+1}{n-1}\right)}}$-indistinguishability operator if and only if it is positive definite.

- A fuzzy relation $E: X \times X \rightarrow[0,1]$ on a set $X$ of odd cardinality $n$ is a $T \frac{1}{\log _{2}\left(\frac{n^{2}+2 n}{n^{2}-2}\right)}$ indistinguishability operator if and only if it is positive definite.

\section{Concluding Remarks}

In this work we have obtained a characterization of positive definite matrices with non-negative entries thanks to the Yager's family of t-norms that gives a surprising application of t-norms to a classical linearalgebraic problem.

We end this work by showing that Propositions 3.5 and 3.6 provide alternative proofs of two important well known facts.

- Since $\min (x, y) \geq T_{\lambda}(x, y)$ for all $\lambda \in(0, \infty)$ and $x, y \in[0,1]$, every min-indistinguishability operator on a finite set is also a $T_{\lambda}$-indistinguishability operator for all $\lambda \in(0, \infty)$. From Proposition 3.5 it follows the next result (see [6] for an alternative proof).

Proposition 4.1. Every min-indistinguishability operator separating points on a finite set is positive definite.

- It is well known that $E$ is a min-indistinguishability operator on a set $X$ if and only if $1-E$ is a pseudoultrametric [8]. By the last proposition, if $E$ separates points, then it is positive definite and therefore $\sqrt{1-E}$ is Euclidean by Corollary 2.8. Since powers of ultrametrics are also ultrametrics, we obtain a new proof of this well-known result ([13]).

Proposition 4.2. Every ultrametric on a finite set is Euclidean.

\section{Acknowledgments}

This research was funded by the Ministerio de Ciencia, Innovacin y Universidades under Grant PGC2018095709-B-C21 and AEI/FEDER, UE funds. 


\section{References}

[1] L.M. Blumenthal, Remark concerning the euclidean four-point property, Ergebnisse Math. Kolloq. Wien 7 (1936) 7-10.

[2] M. Deza, H. Maehara, Metric Transforms and Euclidean Embeddings, Transactions of the American Mathematical Society 317 (1990) 661-671.

[3] S. Gottwald, On t-norms which are related to distances of fuzzy sets BUSEFAL 50 (1992) 25-30.

[4] E. P. Klement, R. Mesiar, E. Pap, Triangular norms, Kluwer, Dordrecht, 2000.

[5] B. Moser, On the T-transitivity of kernels, Fuzzy Sets and Systems 157 (2006) 1787-1796.

[6] B. Moser, On Representing and Generating Kernels by Fuzzy Equivalence Relations, Journal of Machine Learning Research 7 (2006) 2603-2620.

[7] E. Pȩkalska, R.P.W. Duin, The Dissimilarity Representation for Pattern Recognition, Foundations and Applications, World Scientific, Singapore, 2005.

[8] J Recasens, Indistinguishability Operators. Modelling Fuzzy Equalities and Fuzzy Equivalence Relations, Studies in Fuzziness and Soft Computing, Springer, 2011.

[9] J. Recasens, M.S. Tomás, Positive Definite Indistinguishability Operators, Fuzzy Sets and Systems (2019).

[10] I.J. Schoenberg, Metric Spaces and Positive Definite Functions, Transactions of the American Mathematical Society 44 (1938) 522-536.

[11] M.S. Tomás, C. Alsina, J. Rubio-Martinez, Pseudometrics from three-positive semidefinite similarities, Fuzzy Sets and Systems 157 (2006) 23472355 .

[12] L. Valverde, On the Structure of F-indistinguishability Operators, Fuzzy Sets and Systems 17 (1985) 313-328.

[13] I.A. Vestfrid, A.F. Timan, Any separable ultrametric space is isometrically embeddable in $l_{2}$, Funct. Anal. Appl. 17 (1983) 70-73. 